\title{
Erratum to: Rapid transcriptional plasticity of duplicated gene clusters enables a clonally reproducing aphid to colonise diverse plant species
}

Thomas C. Mathers ${ }^{1,3+}$, Yazhou Chen ${ }^{2,3+}$, Gemy Kaithakottil ${ }^{1}$, Fabrice Legeai ${ }^{3,4,5}$, Sam T. Mugford ${ }^{2,3}$, Patrice Baa-Puyoulet ${ }^{3,6}$, Anthony Bretaudeau ${ }^{3,4,5}$, Bernardo Clavijo ${ }^{1}$, Stefano Colella ${ }^{3,6,17}$, Olivier Collin ${ }^{5}$, Tamas Dalmay ${ }^{7}$, Thomas Derrien ${ }^{8}$, Honglin Feng 3,9, Toni Gabaldón 3,10,11,12, Anna Jordan², Irene Julca 3,10,11, Graeme J. Kettles 2,18, Krissana Kowitwanich ${ }^{2,19}$, Dominique Lavenier ${ }^{5}$, Paolo Lenzi ${ }^{2,20}$, Sara Lopez-Gomollon ${ }^{7,21}$, Damian Loska 3,10,11, Daniel Mapleson' ${ }^{1}$, Florian Maumus ${ }^{3,13}$, Simon Moxon', Daniel R. G. Price ${ }^{3,9,22}$, Akiko Sugio ${ }^{2,4}$, Manuella van Munster ${ }^{3,14}$, Marilyne Uzest ${ }^{3,14}$, Darren Waite ${ }^{1}$, Georg Jander ${ }^{3,15}$, Denis Tagu ${ }^{3,4}$, Alex C. C. Wilson ${ }^{3,9}$, Cock van Oosterhout ${ }^{3,16}$, David Swarbreck ${ }^{1,3,16^{*}}$ and Saskia A. Hogenhout ${ }^{2,3,16^{*}}$

\section{Erratum}

After publication of this article [1] we noticed that reference 50 was incorrect. The correct reference 50 is as follows:

Santamaría S, Gonzalez-Cabrera J, Martinez M, Grbic V, Castanera P, Diaz L, Ortego F. Digestive proteases in bodies and faeces of the two-spotted spider mite, Tetranychus urticae. J Insect Physiol. 2015; 78:69-77 http://www.sciencedirect.com/science/article/pii/ S0022191015001018

\footnotetext{
Author details

${ }^{1}$ Earlham Institute, Norwich Research Park, Norwich NR4 7UZ, UK. ${ }^{2}$ John Innes Centre, Norwich Research Park, Norwich NR4 7UH, UK. ${ }^{3}$ The International Aphid Genomics Consortium, Miami, USA. ${ }^{4}$ INRA, UMR 1349 IGEPP (Institute of Genetics Environment and Plant Protection), Domaine de la Motte, 35653 Le Rheu Cedex, France. ${ }^{5}$ RISA/INRIA, GenOuest Core Facility, Campus de Beaulieu, Rennes 35042, France. ${ }^{6}$ Univ Lyon, INSA-Lyon, INRA, BF2I, UMR0203, F-69621 Villeurbanne, France. ${ }^{7}$ School of Biological Sciences, University of East Anglia, Norwich Research Park, Norwich NR4 7TJ, UK. ${ }^{8}$ CNRS, UMR 6290, Institut de Génétique et Developpement de Rennes, Université de Rennes 1 , 2 Avenue du Pr. Léon Bernard, 35000 Rennes, France. ${ }^{9}$ Department of Biology, University of Miami, Coral Gables FL 33146, USA. ${ }^{10}$ Centre for Genomic Regulation (CRG), The Barcelona Institute of Science and Technology, Dr. Aiguader 88, Barcelona 08003, Spain. ${ }^{11}$ Universitat Pompeu Fabra (UPF), 08003 Barcelona, Spain. ${ }^{12}$ Institució Catalana de Recerca i Estudis Avançats (ICREA), Pg. Lluís Companys 23, 08010 Barcelona, Spain. ${ }^{13}$ Unité de Recherche Génomique-Info (URGI), INRA, Université Paris-Saclay, 78026 Versailles, France. ${ }^{14}$ INRA, UMR BGPI, CIRAD TA-A54K, Campus International
}

\footnotetext{
* Correspondence: david.swarbreck@earlham.ac.uk;

saskia.hogenhout@jic.ac.uk

${ }^{\dagger}$ Equal contributors

'Earlham Institute, Norwich Research Park, Norwich NR4 7UZ, UK

${ }^{2}$ John Innes Centre, Norwich Research Park, Norwich NR4 7UH, UK
}

de Baillarguet, 34398 Montpellier Cedex 5, France. ${ }^{15}$ Boyce Thompson Institute for Plant Research, Ithaca, NY 14853, USA. ${ }^{16}$ School of Environmental Sciences, University of East Anglia, Norwich Research Park, Norwich NR4 7TJ, UK. ${ }^{17}$ Present Address: INRA, UMR1342 IRD-CIRAD-INRA-SupAgro-Université de Montpellier, Laboratoire des Symbioses Tropicales et Méditéranéennes, Campus International de Baillarguet, TA-A82/J, F-34398 Montpellier cedex 5, France. ${ }^{18}$ Present address: Rothamsted Research, Harpenden, Hertforshire ALF5 2JQ, UK. ${ }^{19}$ Present address: J. R. Simplot Company, Boise, ID, USA. ${ }^{20}$ Present address: Alson H. Smith Jr. Agriculture and Extension Center, Virginia Tech, Winchester 22602, VA, USA. ${ }^{21}$ Present address: Department of Plant Sciences, University of Cambridge, Downing Street, Cambridge CB2 3EA, UK. ${ }^{22}$ Present address: Moredun Research Institute, Pentlands Science Park, Bush Loan, Penicuik, Midlothian EH26 OPZ, UK.

Received: 29 March 2017 Accepted: 29 March 2017

Published online: 04 April 2017

\section{Reference}

1. Mathers TC, Chen Y, Kaithakottil G, Legeai F, Mugford ST, Baa-Puyoulet P, et al. Rapid transcriptional plasticity of duplicated gene clusters enables a clonally reproducing aphid to colonise diverse plant species. Genome Biol. 2017;18:27. 